\title{
Benefícios dos Exercícios Físicos na Fibromialsia
}

\section{Benefits of Exercise in the Fibromyalgia}

\section{RESUMO}

O exercício é uma intervenção de baixo custo que pode promover saúde em vários aspectos e é capaz de reduzir a dor e outros sintomas da fibromialgia (FM). Nos últimos 20 anos, muitos ensaios clínicos sobre exercício para a FM foram publicados. Apesar dos erros metodológicos, há forte nível de evidência de que exercícios aeróbios supervisionados são eficazes na redução da dor, número de pontos dolorosos, qualidade de vida e depressão. Neste artigo de revisão, os ensaios clínicos de exercício no tratamento da FM são relatados e comentados, e orientações para a prescrição de exercícios foram apresentadas. Foram sugeridos que outros estudos sejam desenvolvidos para melhorar a prescrição de exercício para FM, assim como quais são as principais perguntas a serem respondidas e cuidados metodológicos a serem observados.
\end{abstract}

Valéria Valim $^{(1)}$
Palavras-chave: fibromialgia, exercícios, atividade física.

\section{INTRODUÇÃO}

A síndrome da fibromialgia (FM) se caracteriza por dor crônica generalizada, distúrbio do sono e fadiga ${ }^{(1,2)}$. Estes pacientes, caracteristicamente exibem diminuição da aptidão cardiorrespiratória ${ }^{(3,4)}$. Há evidência de que a atividade física modula a dor em pacientes com FM. Um dos primeiros investigadores a observar relação entre dor e exercício foi Moldofsky ${ }^{(5)}$. Este estudo demonstrou que a privação do sono diminuia o limiar de dor em sedentários, mas o mesmo não acontecia em indivíduos treinados. Apenas uma década mais tarde, ensaios clínicos foram publicados demonstrando que exercícios aeróbios diminuem a dor na $\mathrm{FM}^{(6)}$. A Tabela 1 sintetiza os resultados, desenhos, instrumentos e desfechos dos principais estudos sobre exercício na FM, desde 1988 até 2005.

\begin{abstract}
Exercise is an inexpensive intervention that can improve health in several aspects and it can alleviate chronic pain and other symptoms in fibromyalgia (FM). In the last 20 years many trials have been published. In spite of methodological errors, the evidences strongly support a beneficial role for supervised aerobic exercise training toward reducing pain, number of tender point, quality of life and depression. In this review, the trials on exercise for the fibromyalgia treatment are described and orientations of exercise programs in FM patients are presented. Design and aims of further studies are suggested in order to improve exercise prescription to people with FM.
\end{abstract}

Keywords: fibromyalgia, exercise, physical activity.

Nos últimos 20 anos, foram publicados 28 ensaios clínicos sobre exercício no tratamento da FM, incluindo aproximadamente 1500 pacientes. Desses, 12 avaliaram o condicionamento aeróbio ${ }^{(6-17)}, 10$ estudaram programas combinados ou associados ao tratamento medicamentoso ou outras intervenções não-farmacológicas ${ }^{(18-27)}$.

Apenas três envolveram treino de força ${ }^{(28-30)}$. Exercícios de alongamento foram utilizados como intervenção-controle em muitos estudos ${ }^{(6,10,13,17,29,31)}$.

Uma orientação para a prescrição de exercícios baseada nos trabalhos já descritos ${ }^{(32)}$ e experiência clínica pessoal está apresentada na Tabela 2. Esta tabela está dividida em dois componentes principais: orientações a serem observadas antes do início do programa de exercício, e aquelas durante o programa. 
TABela 1

ENSAIOS CLÍNICOS EM EXERCÍCIO NO TRATAMENTO DA FIBROMIALGIA

\begin{tabular}{|c|c|c|c|c|}
\hline Autor & Ano & $\mathbf{n}$ & Desenho & Resultados/Observações \\
\hline McCain $^{6}$ & 1988 & 42 & AE vs. Flex, ensaio clínico, 20 sem & $\begin{array}{l}\text { Ambos melhoraram } \\
\text { Sem diferença entre grupos }\end{array}$ \\
\hline Mengshoel $^{7}$ & 1992 & 25 & AE vs. Control, ensaio clínico, 20 sem & $\begin{array}{l}\text { Melhora da dor } \\
\text { Fadiga e condicionamento aeróbio não melhoraram }\end{array}$ \\
\hline Isomeri $^{18}$ & 1993 & 45 & Flex + amt vs. ME vs. ME + amt, ensaio clínico, 15 sem & $\begin{array}{l}\text { Associação de exercício com medicação foi superior. } \\
0 \text { programa de exercício não foi descrito }\end{array}$ \\
\hline Nichols ${ }^{8}$ & 1994 & 10 & AE vs. Control & Resultados não-conclusivos \\
\hline Burckhardt ${ }^{19}$ & 1994 & 99 & Educ vs. Control vs. Educ $+\mathrm{AE}$ & $\begin{array}{l}\text { Educação não aumentou a adesão } \\
\text { Nenhuma diferença entre os grupos }\end{array}$ \\
\hline Martin $^{31}$ & 1996 & 60 & ME vs. Relax, ensaio clínico, 6 sem & ME melhorou dor e tender points \\
\hline Wigers $^{9}$ & 1996 & 60 & AE vs. Relax vs. TU, ensaio clínico, 14 sem & $\begin{array}{l}\text { AE melhorou dor, sono, fadiga, depressão } \\
\text { Nenhuma diferença entre os grupos }\end{array}$ \\
\hline Norrergaard ${ }^{10}$ & 1997 & 23 & AE vs. Flex vs. Bolsa quente, ensaio clínico, 12 sem & Qualidade de vida e condicionamento aeróbio não melhoraram \\
\hline Verstappen ${ }^{20}$ & 1997 & 72 & AE vs. Auto ajuda, ensaio clínico, 24 sem & Nenhuma diferença entre os grupos \\
\hline Buckelew $^{21}$ & 1998 & 101 & ME vs. BFB vs. Educ vs. ME + BFB, ensaio clínico, 6 sem & Nenhuma diferença entre os grupos \\
\hline Keel $^{22}$ & 1998 & 27 & $\begin{array}{l}\text { ME + Auto Ajuda vs. Relax, Controlado não-randomizados, } \\
15 \text { sem }\end{array}$ & Nenhuma diferença entre os grupos \\
\hline Gowans $^{23}$ & 1999 & 41 & ME (piscina + solo) vs. Control, ensaio clínico, 6 sem & Dor, sono, fadiga, bem estar melhoraram \\
\hline Ramsay $^{24}$ & 2000 & 74 & $\begin{array}{l}\text { AE supervisionado vs. Não supervisionado, ensaio clínico, } \\
\qquad 12 \mathrm{sem}\end{array}$ & Supervisionado foi superior quanto aos aspectos psicológicos \\
\hline Jones $^{29}$ & 2000 & 63 & Força vs. Flex, ensaio clínico, 12 sem & Nenhuma diferença entre os grupos \\
\hline Meyer $^{42}$ & 2000 & 8 & AE Alta vs. Baixa Intensidade, piloto, 8 sem & Nenhuma diferença entre os grupos \\
\hline Jentoft ${ }^{11}$ & 2001 & 47 & Piscina vs.Solo AE, ensaio clínico, 20 sem e 6 meses & Nenhuma diferença entre os grupos \\
\hline Gowans $^{12}$ & 2001 & 57 & Piscina vs. Solo (caminhada), ensaio clínico, 23 sem & Nenhuma diferença entre os grupos \\
\hline Hakkinen $^{28}$ & 2001 & 21 & Força vs. Control, ensaio clínico, 21 sem & Nenhuma diferença entre os grupos \\
\hline Mannerkorpi ${ }^{25}$ & 2002 & 58 & Piscina vs. Educ, ensaio clínico, 24 sem 48 meses & $\begin{array}{l}\text { Dor, Sono, fadiga, qualidade de vida, depressão, ansiedade, condicionamento } \\
\text { foram superiores }\end{array}$ \\
\hline $\mathrm{Geel}^{30}$ & 2002 & 10 & Força, não controlado, 8 sem & Treino de força é seguro \\
\hline Rooks $^{27}$ & 2002 & 15 & Força vs. ME, ensaio clínico, 20 sem & Treino de força é seguro \\
\hline Van Santen ${ }^{14}$ & 2002 & 37 & AE Alta vs. Baixa, ensaio clínico, 20 sem & Ambos não melhoraram. Alta intensidade aumentou a dor \\
\hline Van Santen ${ }^{26}$ & 2002 & 143 & BFB vs. ME vs. TU, ensaio clínico, 24 sem & Nenhuma diferença entre os grupos \\
\hline Richards $^{13}$ & 2002 & 136 & AE vs. Flex, ensaio clínico, 12 & AE melhorou tender points \\
\hline King ${ }^{15}$ & 2002 & 152 & $\begin{array}{l}\text { AE vs. } A E+\text { Educ vs. Educ vs. Control, ensaio clínico, } 12 \\
\text { sem }\end{array}$ & Nenhuma diferença entre os grupos \\
\hline Schachter ${ }^{16}$ & 2003 & 143 & Curto vs. longo AE vs. control & Nenhuma diferença entre os grupos \\
\hline Valim $^{17}$ & 2003 & 76 & AE vs. Flex, ensaio clínico, 20 sem & $\begin{array}{l}\text { AE foi superior a Flex para melhora da qualidade de vida e depressão. Não houve } \\
\text { correlação entre ganhar condicionamento e aumentar qualidade de vida }\end{array}$ \\
\hline Valim $^{43}$ & 2003 & 20 & AE Alto vs. Baixa intensidade, ensaio clínico, 20 sem & Nenhuma diferença entre os grupos \\
\hline Assis $^{36}$ & 2005 & 60 & Piscina vs. solo $A E$, ensaio clínico, 15 sem & $\begin{array}{l}\text { In press Deep water running é superior a caminhada na melhora do FIQ e } \\
\text { aspectos psicológicos }\end{array}$ \\
\hline
\end{tabular}

$\mathrm{AE}=$ Exercício aeróbio; Flex = Exercício de Flexibilidade; sem = semanas; amt = amitriptilina; $\mathrm{ME}=$ exercícios mistos (combinados); Educ = Educação; Relax = Relaxamento; Control = grupo-controle; $\mathrm{TU}=$ tratamento usual; $\mathrm{BFB}=$ biofeedback; Força = Exercícios de fortalecimento 
TABELA 2

ORIENTAÇÕES PARA PROGRAMAS DE EXERCíCIO AERÓBIO EM PACIENTES COM FIBROMIALGIA

1- $\quad$ Antes de iniciar um programa de atividade física a. Avaliar risco cardiovascular

b. Avaliar e tratar co-morbidades musculoesqueléticas

c. Avaliação funcional

d. Checar medicações em uso

e. Perguntar sobre história pregressa de atividade física

f. Quando o uso de medicamentos para fibromialgia estiver indicado, iniciá-lo quatro semanas antes de começar a atividade física para melhorar a dor e tolerância ao esforço

g. Explicar a importância do exercício no tratamento

h. Explicar que deverá ser "para sempre"

i. Preparar o paciente para uma possível piora da dor nas primeiras oito semanas de treinamento

j. Explicar aos pacientes que os benefícios ocorrem após 8-10 semanas e continuam aumentando até 20 semanas.

a. Individualize a prescrição

b. Tipo de exercício: Condicionamento aeróbio deve ser prescrito para todos, exceto quando houver condição associada que o contra-indique. Treinos de força e alongamento são seguros e podem ser prescritos se houver contraindicação para treino aeróbio ou por preferência do paciente.

c. A preferência do paciente deve ser considerada na escolha da modalidade.

d. Modalidade: de baixo impacto

e. Intensidade: Treinamento na frequência cardíaca do limiar anaeróbio. Quando fórmulas forem utilizadas, prescrever $65-70 \%$ da $\mathrm{FC}_{\text {max }}$, ou $50-55 \%$ da FCR (Karvonen).

f. Iniciar com carga menor e aumentar progressivamente (treino escalonado)

g. Diminuir carga quando houver piora sintomática

h. Evitar super treinamento

i. Minimizar contrações excêntricas

j. Se possivel, oferecer programa supervisionado ou atividades em grupo

k. Estimular pacientes a participar de grupos e associações (de pacientes)

l. Ser otimista sobre o quanto o exercício poderá ajudá-los

m. Prescrição por escrito
Antes de iniciar um programa de exercícios é importante fazer uma avaliação funcional e cardiovascular para identificar condições que possam interferir no desempenho e na resposta ao exercício ou oferecer risco como doença coronariana e hipotensão postural. As co-morbidades musculoesqueléticas podem limitar o treinamento e devem ser tratadas previamente. Atenção especial deve ser dada à revisão dos medicamentos em uso, pois muitos interferem na resposta hemodinâmica. A anamnese também deve conter informações da história pregressa de hábito de atividade física (freqüência, modalidade, preferência, tolerância e comportamento familiar em relação ao exercício). Estas informações ajudam a individualizar a prescrição e aumentar a adesão. Vale também reforçar o quanto o exercício é importante no controle da dor e de vários sintomas relacionados. Importante informar que embora deva ser praticado indefinidamente, $\mathrm{o}$ benefício ocorre apenas entre oito e dez semanas após o início do programa e continua aumentando até a vigésima semana, mas alguns indivíduos podem sentir-se pior e com mais dor, inicialmente ${ }^{(17)}$.

Assim como a prescrição de medicamentos deve conter dose, duração e intervalo, a prescrição do exercício deve detalhar orientações sobre a intensidade inicial do treino e como aumentar progressivamente a carga. Para adequada prescrição individual é importante considerar as preferências do paciente, co-morbidades, uso de medicamentos, capaci- 
dade funcional e se possível, avaliação ergométrica.

Sugerimos que a prescrição do exercício seja por escrito, pelo médico, como é feito com os medicamentos, pois isto aumenta a adesão e facilita a interface com os fisioterapeutas e educadores físicos. A relação médico-paciente pode e deve ser usada para aumentar a adesão também nas intervenções não-farmacológicas.

Mais de 60 diferentes instrumentos de avaliação têm sido usados em estudos sobre exercícios e FM, dificultando a meta-análise. Muitos não descreveram os protocolos de exercício com informação suficiente sobre intensidade, duração, freqüência e modalidade usadas nos grupos intervenção-controle ou intervenção. Efeitos adversos também têm sido pobremente relatados ${ }^{(33)}$. Apesar das falhas metodológicas de alguns estudos, há forte evidência de que o exercício aeróbio supervisionado reduz a dor, o número de pontos dolorosos, depressão, ansiedade, melhora a qualidade de vida, e outros aspectos psicológicos ${ }^{(17,33)}$. Assim, atividade física aeróbia deve ser prescrita para todos os pacientes com FM, com exceção daqueles com alguma contra-indicação (raramente).

Enquanto o benefício mais expressivo parece ser com exercícios aeróbios, o fortalecimento e o alongamento também têm efeitos terapêuticos. $\mathrm{O}$ alongamento tem sido usado como intervenção-controle, mas não é um placebo ideal, pois foi observada alguma melhora. É interessante observar que os aspectos emocionais e psicológicos foram modificados pelo exercício aeróbio, mas não pelo alongamento. Quando os componentes físicos e psicológicos foram agrupados, ficou claro que o condicionamento aeróbio é superior ao alongamento ${ }^{(17)}$. Uma hipótese para explicar esta observação é que o treino aeróbio provoca mudanças neuroendócrinas necessárias para a melhora do humor (aumento de serotonina e norepinefrina) e o alongamento não.

Poucos estudos, com amostra pequena sobre fortalecimento na FM, têm sido publicados e demonstraram que o treino de força promove maior melhora quando comparado com treino de flexibilidade ${ }^{(28-30)}$. Mais estudos comparando treino de força e alongamento são necessários, mas ambos são seguros e podem ser prescritos.

Assim como para outras doenças reumáticas, a hidroterapia é comumente prescrita na FM. Existem seis estudos publicados avaliando balneoterapia, banhos e exercícios aeróbios na água ${ }^{(11,23,25,34-36)}$. Assis e cols ${ }^{(36)}$ recentemente demonstraram que deep running é um pouco melhor que o condicionamento aeróbio em solo na melhora dos escores do Fibromyalgia Impact Questionnaire (FIQ) e aspectos psicológicos da qualidade de vida.
Pacientes com FM parecem necessitar de um período maior e mais esforço pessoal para adaptação a um programa de exercício. Por isso, a progressão da carga deve ser mais lenta que o habitual. Além disso, pacientes podem até piorar nas primeiras oito semanas ${ }^{(6)}$. Por isso, ensaios de curta duração não demonstraram melhora na qualidade de $\operatorname{vida}^{(19,21,23,31)}$, enquanto programas mais longos, com mais de 15 semanas conseguiram observar melhora em vários aspectos, inclusive qualidade de vida ${ }^{(17,25,26)}$.

Uma outra observação interessante nesta população é que os benefícios conseqüentes do alongamento ocorrem em dez semanas e se estabilizam, enquanto a melhora associada ao condicionamento aeróbio é evidente com dez semanas e continua aumentando até 20 semanas. Então, o tempo necessário para melhora sintomática pode ser diferente para cada tipo de exercício. A análise dos resultados no tempo ( 10 e 20 semanas) mostra que os pacientes primeiro ganham aptidão física e apenas depois ocorre melhora clínica ${ }^{(17)}$.

Nos ensaios clínicos publicados sobre exercício na FM, as perdas variaram de 9 a $50 \%{ }^{(32)}$. Esta baixa adesão ao exercício também ocorre em populações saudáveis. Apenas $8 \%$ dos homens e $2 \%$ das mulheres entre 50 e 64 anos estão engajados em uma atividade física regular ${ }^{(37)}$. Apesar dos benefícios à saúde, apenas $50 \%$ das pessoas saudáveis que iniciam um programa de exercício continuam após seis meses $^{(38)}$. Na realidade, a baixa adesão não é um problema exclusivo das intervenções físicas. Apenas $30 \%$ dos pacientes retornam para reavaliação e apenas $50 \%$ tomam a medicação prescrita. Em pacientes com FM, os fatores mais preditivos de adesão a um programa regular de exercício foram idade, nível socioeconômico, depressão, escolaridade, auto-eficácia e convívio social ${ }^{(39)}$. Em um estudo transversal ${ }^{(40)}$, interação com outros pacientes com FM também é importante, pois o principal fator relacionado ao nível de atividade física foi ser membro de uma associação de pacientes. Apenas dois estudos foram desenhados para avaliar os determinantes de adesão, mostrando que um programa educacional associado não aumentou a participação regular ${ }^{(15)} \mathrm{e}$ que exercícios supervisionados $^{(24)}$ têm maior adesão $(72 \%)$ do que treino não-supervisionado $(50 \%, \mathrm{p}<0.05)$. Os indivíduos que abandonaram o tratamento foram aqueles que inicialmente tinham piores escores de dor, eram mais estáveis emocionalmente e com maior vitalidade. Então, indivíduos com mais dor podem ter menos tolerância ao esforço, e aqueles que se sentem emocionalmente piores podem sentir-se mais apoiados e cuidados em um grupo. Neste mesmo estudo, a adesão foi similar no grupo de alongamento e de treino aeróbio $^{(17)}$. 
De acordo com Jones et al ${ }^{32)}$ : "Embora por décadas é conhecido que o exercício é um componente-chave no tratamento da FM, a maioria dos pacientes permanece sedentária“. A não-adesão a um programa de exercício é percebida pelos profissionais de saúde como um desejo inconsciente de permanecer doente. Por esta razão, alguns médicos condicionam o tratamento com a adesão à atividade física. Uma melhor compreensão das variáveis envolvidas no exercício irá permitir melhor abordagem e adesão.

Apenas cinco estudos usaram parâmetros de avaliação e prescrição definidos de acordo com o American College of Sports Medicine (ACSM). Os critérios de prescrição de intensidade de treino aeróbio aceitos pelo ACMS são a freqüência cardíaca máxima $\left(\mathrm{FC}_{\max }\right)$, freqüência cardíaca de reserva (FCR) ou consumo de reserva de oxigênio $\left(\mathrm{VO}_{2} \mathrm{R}\right)$ e limiar anaeróbio (LA). A FC ${ }_{\text {max }}$ pode ser obtida por teste ergométrico ou freqüência cardíaca estimada para a idade. $\mathrm{O}$ limiar anaeróbio pode ser definido como o maior consumo de oxigênio obtido sem acidose lática sustentada. Pode ser medido por lacticemia ou por análise de gás expirado. Para ser considerado um treino aeróbio, o indivíduo tem que atingir $55 \% / 65-90 \%$ da $\mathrm{FC}_{\max }$ ou $40 \% / 50-85 \%$ do $\mathrm{VO}_{2} \mathrm{R}$ ou da FCR. Os níveis mais baixos de intensidade de treino, por exemplo $40-49 \%$ da $\mathrm{VO}_{2} \mathrm{R}$ ou FCR, e $55-64 \%$ da $\mathrm{FC}_{\max }$, são os mais aplicáveis para indivíduos descondicionados ${ }^{(41)}$.

A intensidade de treinamento ideal não está estabelecida. Em dois estudos prévios ${ }^{(17,36)}$ não houve correlação entre o nível de condicionamento cardiorrespiratório atingido e a melhora na qualidade de vida dos participantes, sugerindo que os programas de exercícios não precisam estar focados em ganhar aptidão física. No entanto, nem sempre aquilo que parece óbvio se comprova ser verdadeiro. Atualmente, o ACMS já reconhece que a intensidade de treinamento para promoção de saúde (reduçãodo risco coronariano, melhora metabólica e de doenças degenerativas) é menor do que aquela necessária para aumentar a aptidão cardiorrespiratória traduzida por aumento de consumo de oxigênio. Entretanto, é importante conhecer a intensidade mínima de treino capaz de promover melhora da dor.

Três estudos abordaram esta questão, todos tiveram amostra pequena e falharam em apresentar diferença significativa entre grupos de alta e baixa intensidades ${ }^{(14,42,43)}$. Considerando estes resultados e que pacientes com FM são descondicionados, o consenso atual é de que a prescrição de treino de baixa intensidade é mais adequada ${ }^{(32)}$. Por outro lado, estudos que utilizaram apenas baixa intensidade mostraram tendência de melhora, mas falharam em demonstrar benefícios estatisticamente signifitivos. Analisando os estudos, os três que prescreveram programa de moderada a alta intensidades, definido como aquele capaz de elevar a freqüência cardíaca até a freqüência do limiar anaeróbio, foram os que conseguiram benefícios clínico e estatístico mais significativos ${ }^{(17,6,36)}$. É possível que treinos de moderada a alta intensidades sejam os mais efetivos em reduzir dor e sintomas psicoafetivos em pacientes com FM.

Considerando que pacientes com FM geralmente não atingem teste máximo, o limiar anaeróbio é o melhor parâmetro de prescrição e avaliação de aptidão cardiorrespiratória e deveria ser utilizado em todos os protocolos de pesquisa ${ }^{(4)}$. Entretanto, para sua obtenção é necessário análise do gás expirado, que não pode ser realizado em todos os centros. Recomendamos que na FM, a intensidade de treinamento seja a freqüência cardíaca do LA ou logo abaixo dela, pois indica uma intensidade adequada para ganho de aptidão, com maior segurança e maior adesão. Infelizmente, a realização de teste ergoespirométrico para determinação do LA não é acessível para a maioria, na prática clínica diária. Quando possível, a determinação do LA indica a intensidade de treino ideal (tolerável e capaz de promover melhora clínica significativa).

Em pacientes com FM, a freqüência cardíaca do LA é mais baixa do que nos controles normais ${ }^{(4)} \mathrm{e}$, diferente do que seria o esperado, a FCmax e a de repouso não mudam depois de adequado treino aeróbio ${ }^{(17)}$. Então, quando não for possível medir diretamente o LA, recomendamos que em pacientes com FM, a fórmula de Karvonen seja usada para encontrar a freqüência de treino, pois ela considera a $\mathrm{FC}_{\max }$ e a de repouso. $\mathrm{A} \mathrm{FC}_{\max }$ pode ser estimada pelas fórmulas [220-idade] e [208 - 0,7* idade]. Assim, a $\mathrm{FC}_{\max }$ estimada pode ser aplicada na fórmula de Karvonen, conforme exemplificado a seguir: FC de treino $(60-80 \%)=[(\mathrm{FCmax}$ - FCR $) * 60 \%$ FCmax + FCR]. Porém, de um modo geral, um inconveniente para a prescrição de exercícios baseados em fórmulas é a grande variabilidade da frequiência cardíaca entre os indivíduos, especialmente para aqueles que têm fibromialgia, já que podem apresentar resposta cronotrópica anormal ao exercício por disautonomia. Estudos futuros são necessários para conhecer a resposta cronotrópica ao esforço em pacientes com FM com e sem disautonomia e saber se estas fórmulas podem ser validadas e aplicadas em pacientes com FM ou se há fórmula específica para esta população.

Com base nos estudos apresentados, as perguntas que ainda precisam ser respondidas bem como algumas observações para melhorar a qualidade metodológica dos estudos futuros sobre exercício na FM estão apresentados nas Tabelas 3 e 4. 
a. Programas de fortalecimento muscular são melhores, iguais ou inferiores ao condicionamento aeróbio?

b. Os programas de alta intensidade são mais eficientes na melhora clínica que os de baixa intensidade?

c. Quais sintomas melhoram com qual tipo de exercício? Há sintomas que os exercícios não podem melhorar? Qual benefício devemos esperar de cada tipo de exercício?

d. Qual o impacto do exercício nos diferentes domínios da qualidade de vida em pacientes com FM?

e. Outros investigadores podem reproduzir o relato inicial de que o exercício aeróbio pode melhorar sintomas de depressão em pacientes com FM?

f. Há subtipos de pacientes com FM que respondem melhor ao exercício?

g. As fórmulas de prescrição de exercício baseadas na frequência cardíaca são válidas e comparáveis ao LA em pacientes com FM?

h. Como é a resposta cronotrópica ao exercício em pacientes com FM que têm disautonomia?

i. Os fatores abaixo determinam maior adesão ao exercício:

- $\quad$ Programas de educação que motivem a atividade física

- Supervisão por um paciente treinado ou por um membro da família

- Intensidade do treino

- Uso de piridostigmina ou medicação analgésica?

TABELA 4

SugESTÕES PARA MELHORAR A QUALIDADE METOLÓGICA DOS PRÓXIMOS ESTUdOS
a. Descrever detalhadamente os protocolos de exercício incluindo intensidade, duração e frequência;
b. Descrever eventos adversos;
c. Usar parâmetros de avaliação e prescrição validados e reconhecidos pelo ACSM;
d. Definir um número limitado de variáveis a serem estudadas, escolhendo preferencialmente instrumentos já validados ou testados na FM, tais como: EVA, FIQ, SF-36, Beck Depression Index ;
e. Ser longo o suficiente para que se atinja todos os efeitos terapêuticos (mínimo de 15 semanas);
f. Tratar previamente outras condições clínicas que possam interferir no desempenho no exercício (ex. osteoartrite, dor miofacial, lesão de partes moles, distúrbio do equilíbrio). Alternativamente, deve-se analisar esta variável separadamente ou excluir pessoas com estas limitações.

\section{REFERÊNCIAS}

1. Moldofsky H: Sleep and fibrositis syndrome. Rheum Dis Clin North Am 5: 91-103, 1989.

2. Wolfe F, Smythe HA, Yunus MB et al: The American College of Rheumatology 1990 criteria for classification of fibromyalgia: report of the multicenter criteria committee. Arthritis Rheum 33: 160-72, 1990.

3. Bennet RM, Clark SR, Goldenberg L: Aerobic fitness in patients with fibromyalgia - a controlled study of respiratory gas exchange and $133 x$ xenon clearence from exercising muscle. Arthritis Rheum 32: 454-60, 1989

4. Valim V, Oliveira LM, Suda AL et al: Peak oxygen uptake and ventilatory anaerobic threshold in fibromyalgia. J Rheumatol 29: 353-7, 2002.
5. Moldofsky H, Scarisbrick P: Induction of neurasthenic musculoskeletal pain syndrome by selective sleep stage deprivation. Psychosom Med 38: 35-44, 1976.

6. McCain GA, Bell DA, Mai FM et al: A controlled study of the effects of a supervised cardiovascular fitness training program on the manifestations of primary fibromyalgia. Arthritis Rheum 31: 1135-41, 1988.

7. Mengshoel AM, Komnaes HB, Forre O: The effects of 20 weeks of physical fitness training in female patients with fibromyalgia. Clin Exp Rheumatol 10: 345-9, 1992.

8. Nichols DS, Glenn TM: Effects of aerobic exercise on pain perception, affect, and level of disability in individuals with fibromyalgia. Phys Ther 74: 372-32, 1994.

9. Wigers SH, Stiles TC, Vogel PA: Effects of aerobic exercise versus stress management treatment in fibromyalgia - a 4.5 year 
prospective study. Scand J Rheumatol 28: 1056-62, 1996.

10. Norregaard J, Lykkegaard JJ, Mehlsen J, Danneskiold-Samsoe B: Exercise training in treatment of fibromyalgia. J Musculoskeletal Pain 5: 71-9, 1997.

11. Jentoft ES, Kvalvik AG, Mengshoel AM: Effects of pool-based and land-based aerobic exercise on women with fibromyalgia/ chronic widespread muscle pain. Arthritis Rheum 45: 42-7, 2001.

12. Gowans SE, deHueck A, Voss S, Silaj A, Abbey SE, Reynolds WJ: Effect of a randomized, controlled trial of exercise on mood and physical function in individuals with fibromyalgia. Arthritis Rheum 45: 519-29, 2001.

13. Richards SCM, Scott DL: Prescribed exercise in people with fibromyalgia parallel group randomised controlled trial. BMJ 325: 185-7, 2002.

14. Van Santen M, Bolwijn P, Verstappen F et al: High or low intensity aerobic fitness training in fibromyalgia: does it matter? J Rheumatol 29: 582-7, 2002.

15. King SJ, Wessel J, Bhambhani $Y$ et al: The effects of exercise and education, individually or combined, in women with fibromyalgia. J Rheumatol 29: 2620-7, 2002.

16. Schachter CL, Bush AJ, Peloso PM, Sheppard MS: Effects of short versus long bouts of aerobic exercise in sedentary women with fibromyalgia: a randomized controlled trial. Phys Ther 83: 340-58, 2003.

17. Valim V, Oliveira LM, Suda AL et al: Aerobic fitness effects in fibromyalgia. J Rheumatol 30: 1060-9, 2003.

18. Isomeri $\mathrm{R}$, Mikkelsson $\mathrm{M}$, Latikka P et al: Effects of amitriptyline and cardiovascular fitness training on pain in patients with primary fibromyalgia. J Musculoskeletal Pain 1: 253-60, 1993.

19. Burckhardt CS, Mannerkorpi D, Hedenberg L et al. A randomised controlled clinical trial of education and physical training for women with fibromyalgia 1994. J Rheumatol 21: 714-20, 1994.

20. Verstappen FTJ, Santen-Hoeuftt HMS, Bolwijn PH et al: Effects of a group activity program for fibromyalgia patients on physical fitness and well being. J Musculoskeletal Pain 5: 17-28, 1997.

21. Buckelew SP, Conway R, Parker J et al: Biofeedback/relaxation training and exercise interventions for fibromyalgia. Arthritis Care Res 11:196-209, 1998.

22. Keel PJ, Bodoky C, Gerhard U et al: Comparison of integrated group therapy and group relaxation training for fibromyalgia. Clin J Pain 14: 232-8, 1998.

23. Gowans SE, Dehueck A, Voss S et al : A randomised controlled trial of exercise and education for individuals with fibromyalgia. Arthritis Care Res 12: 120-8, 1999.

24. Ramsay C, Moreland J, Ho M et al: An observer-blinded comparison of supervised and unsupervised aerobic exercise regimens in fibromyalgia. Rheumatology 39: 501-5, 2000.

25. Mannerkorpi K, Burckhardt CS, Bjelle A: Pool exercise combined with an education program for patients with fibromyalgia syndrome. A prospective randomised study. Scand J Rheumatol 31: 306-10, 2002.

26. Van Santen M, Bolwijn P, Verstappen F et al: A randomized clinical trial comparing fitness and biofeedback training versus basic treatment in patients with fibromyalgia. J Rheumatol 29:
575-81, 2002.

27. Rooks DS, Silverman CB, Kantrowitz FG: The effects of progressive strength training and aerobic exercise on muscle strength and cardiovascular fitness in women with fibromyalgia: a pilot study. Arthritis Care Res 47: 22-8, 2002.

28. Häkkinen A, Häkkinen $\mathrm{K}$, Hannonen $\mathrm{P}$ et al: Strength training induced adaptations in neuromuscular function of premenopausal women with fibromyalgia: comparison with healthy women. Ann Rheum Dis 60: 21-6, 2001.

29. Jones KD, Burckhardt CS, Clark SR, Bennett RM, Potempa KM: A randomised controlled trial of muscle strengthening versus flexibility training in fibromyalgia. J Rheumatol 29: 1041-8, 2002.

30. Geel S, Robergs RA: The effect of graded resistance exercise on fibromyalgia symptoms and muscle bioenergetics: a pilot study. Arthritis Care Res 47: 82-6, 2002.

31. Martin L, Nutting A, Macintosh BR et al: An exercise program in the treatment of fibromyalgia. J Rheumatol 23: 1050-3, 1996.

32. Jones $\mathrm{KD}$, Clarck SR: Individualizing the exercise prescription for persons with fibromyalgia. Rheum Dis Clin North Am 28: 419-36, 2002.

33. Busch A, Schachter CL, Peloso PM, Bombardier C: Exercise for treating fibromyalgia syndrome. Cochrane Database Systematic Review 2, 2005.

34. Günther V, Mur E, Kinigadner Uet al: Fibromyalgia - effect of relaxation and hydrogalvanic bath therapy on the subjective pain experience. Clin Rheumatol 13: 573-8, 1994.

35. Evcik D, Kizilay B, Gokcen E: The effects of balneotherapy on fibromyalgia patients. Rheumatol Int. 22: 56-9, 2002.

36. Assis MR, Silva LE, Alves A, Pessanha AP, Feldman D, Barros Neto TL, Natour J: Deep water running - an aquatic exercise to treat fibromyalgia: a randomized, controlled study - Arthritis Rheum 48: S303, 2003.

37. Sallis JF, Haskell WL, Wood PD et al: Physical activity assessment methodology in the five-city project. Am J Epidemiol 121: 91106, 1985.

38. Dishman, RK: Compliance/adherence in health related exercise. Health Psychol 1: 237-67, 1982.

39. Oliver K: Predictors of exercise behaviors among fibromyalgia patients. Prev Med 35: 383-9, 2002.

40. Natvig B, Bruusgaard D, Eiksen W: Physical leisure activity level and physical fitness among women with fibromyalgia. Scand J Rheumatol 27: 337-41, 1998.

41. Pollock ML, Gaesser GA, Butcher JD et al. The recommended quantity and quality of exercise for developing and and maintaining cardiorespiratory and muscular fitness, and flexilibility in healthy adults. Med Sci Sport Exe 30: 975-91, 1998.

42. Meyer BB, Lemley KJ: Utilizing exercise to affect the symptomatology of fibromyalgia: a pilot study. Med Sci Sports Exerc 32: 1691-7, 2000.

43. Valim V, Coelho PF, Caldeira CT: Ensaio Clínico Controlado Randomizado comparando os efeitos do treinamento aeróbio de alta intensidade com o de baixa intensidade em pacientes com fibromialgia. Jornada Brasileira de Reumatologia. Porto de Galinhas, Pernambuco, 2003. 